\title{
La promotion hiérarchique et la compétence en communication
}

André Lafrance

\section{OpenEdition}

1 Journals

Édition électronique

URL : http://journals.openedition.org/communicationorganisation/1704

DOI : 10.4000/communicationorganisation. 1704

ISSN : $1775-3546$

Éditeur

Presses universitaires de Bordeaux

Édition imprimée

Date de publication : 1 mai 1994

ISSN : 1168-5549

Référence électronique

André Lafrance, «La promotion hiérarchique et la compétence en communication », Communication et organisation [En ligne], 5 | 1994, mis en ligne le 26 mars 2012, consulté le 30 avril 2019. URL : http:// journals.openedition.org/communicationorganisation/1704; DOI : 10.4000/ communicationorganisation. 1704

Ce document a été généré automatiquement le 30 avril 2019

(c) Presses universitaires de Bordeaux 


\title{
La promotion hiérarchique et la compétence en communication
}

\author{
André Lafrance
}

1 On juge souvent la compétence d'un gestionnaire d'après celle de ses collaborateurs. Car leur degré de compétence sera un facteur important dans la réussite de ses projets. Il a donc tout intérêt à bien les choisir. Et c'est justement le problème ! Comment être sûr que la personne qu'on a l'intention de promouvoir à un poste de plus grande responsabilité saura faire preuve de la compétence attendue ? Certains observateurs iront même jusqu'à dire que c'est une tâche impossible. Ils invoqueront souvent, comme base de leur pessimisme, le célèbre Principe de Peter. Lancé il y a 23 ans, cet ouvrage a été un des plus grands succès de librairie: plus de huit millions d'exemplaires vendus en 37 langues! Dans un texte marqué d'un humour parfois acerbe qui reprenait le style rendu célèbre par la Loi de Parkinson, l'auteur semblait avoir formulé une des lois fondamentales des organisations humaines : « dans une hiérarchie, un employé tend à s'élever à son niveau d'incompétence ». Le magazine LIFE écrivait que cette petite phrase était devenue un phénomène culturel et qu'elle allait, dorénavant, faire partie du discours sur le management.

\section{Une explication universelle}

2 Comment expliquer un tel succès? Dans son livre sur Les grands auteurs en organisation, Jean-Claude Scheid consacre un chapitre à Parkinson et Peter dans la section « auteurs et théories sociologiques des organisations ». Il croit qu »'on peut voir dans leurs écrits si connus de par le monde, la traduction d'un réflexe populaire de méfiance à l'égard de la toute puissance des grandes organisations » (4).Mais cela n'explique pas que le Principe de Peter ait trouvé sa plus grande utilisation au sein même des organisations.

3 Nous pensons plutôt que son postulat liant incompétence et promotion hiérarchique s'insère bien dans la lignée des explications qui mettent l'accent sur les individus plutôt que sur les structures ou les modalités de gestion/production. «Nous avons un bon 
produit, nous offrons de bons services et nous avons une entreprise qui fonctionne bien. Alors pourquoi rencontrons-nous tant de problèmes? » Il serait trop inconfortable de mettre en cause la base même de l'édifice garantissant la situation actuelle des intervenants. Aucun d'entre eux n'est prêt à « couper la branche sur laquelle il est assis ». Donc, comme cela ne peut être la faute de l'organisation, il faut identifier des coupables qui empêchent cette dernière de fonctionner aussi bien qu'elle le devrait.

4 La solution : c'est la faute de tout le monde et de personne. Tout le monde atteint son niveau d'incompétence; mais personne ne peut rien y faire puisque c'est un principe universel. Si on vous trouve compétent, vous ne perdez rien pour attendre qu'on vous place en position d'être incompétent. En somme c'est votre compétence même qui vous met en péril d'incompétence.

5 En vertu de ce principe, le cadre supérieur se voit donc confronté à un dilemme: promouvoir un collaborateur à son niveau d'incompétence ou accepter de ne pas utiliser la totalité de sa compétence en ne lui confiant pas toutes les responsabilités qu'il serait capable d'assumer. Toute promotion devient un " coup de dé »; on s'y lance en espérant que le destin oubliera, pour cette fois, les effets désastreux du Principe omniprésent.

6 On constate pourtant que les membres d'une organisation peuvent jouir d'un certain «libre-arbitre" face au destin "diabolique" de la promotion. On a vu des cadres s'adapter à leur nouvelle fonction et y trouver le stimulant nécessaire pour développer une compétence dans l'exercice de responsabilités plus larges. Le Principe de Peter n'est donc pas inéluctable. Mais comment évaluer les risques et, surtout, comment les diminuer?

\section{La composante humaine}

7 Le mauvais fonctionnement d'un cadre n'est pas nécessairement dû à une poussée d'incompétence comme s'il s'agissait d'une fièvre causée par un nouveau climat de responsabilités. C'est trop facile d'excuser une mauvaise planification stratégique par une accumulation d'incompétences opérationnelles. Il y a là comme une sorte de syndrome de Chernobyl, reprenant les premières réactions officielles à la suite de ce terrible accident. Ce syndrome s'exprime par la position suivante : personne ne doit officiellementmettre en doute le principe de construction d'une centrale nucléaire dont la sécurité ne peut qu'avoir été trahie par un mauvais fonctionnement...de la composante humaine. Car il est plus rentable à court terme de focaliser la vindicte populaire sur une douzaine de «responsables» que d'impliquer les nombreuses filières de responsabilités dans la conception, l'implantation et l'organisation de ces installations. En fustigeant l'incompétence de ces "boucs émissaires", on cautérise une source potentielle d'hémorragie dans la confiance publique.

8 Nous n'allons sûrement pas sous estimer l'importance de cette composante humaine. Mais il serait malheureux que la présomption d'incompétence entraîne les cadres supérieurs d'une organisation à mettre en place des mécanismes d'encadrement ou de suppléance qui limitent l'initiative de leurs collaborateurs. Car c'est dans cette marge de manœuvre que résident, pour eux, les occasions de démontrer leur compétence. L'incompétence appréhendée a une fâcheuse tendance à se réaliser lorsqu'on retire des mains de la « victime » tous les instruments qui pourraient lui permettre de lutter contre cette nouvelle forme d'entropie personnelle. On pourrait appliquer au changement 
individuel ce que Philippe Bernoux, à la suite de Michel Crozier, présente comme conditions indispensables au changement organisationnel. "Tout changement est accepté dans la mesure où l'acteur pense qu'il a des chances de gagner quelque chose et, en tout cas, sent qu'il maîtrise suffisamment les leviers et les conséquences du changement. Celui-ci doit se raisonner en termes de pouvoir : celui qui a le sentiment de perdre ne peut que refuser le changement ou tenter de le freiner ». À plus ou moins long terme, l'absence de ces conditions crée une sorte de déficience immunitaire qui empêche le cadre promu de résister au stress imposé par ses nouvelles fonctions.

\section{Le stress et l'incompétence appréhendée}

Empruntant à la dynamique des métaux, on a d'abord identifié l'existence d'un stress humain causé par une réponse physiologique du corps à un changement. Mais on a pris un certain temps à comprendre que l'apparition d'un changement n'entraînait pas nécessairement les effets pernicieux d'un stress, par ailleurs indispensable à la focalisation des énergies de l'acteur impliqué dans le changement. Les travaux de Richard S. Lazarus ont démontré que le stress ne devenait handicapant qu'au moment où cet acteur se percevait comme incapable de faire face au changement.

Si l'organisation a intégré dans sa culture la présomption d'incompétence (même temporaire) associée à la promotion, elle contribue, même involontairement, à développer chez ses membres des conditions propices à cette perception de leurs propres situations. Elle génère les effets négatifs du stress organisationnel et confirme, chez le nouveau cadre, la conviction qu'il ne pourra assumer adéquatement ses nouvelles responsabilités. Et l'hypothèse fondamentale du Principe de Peter en arrive à provoquer sa propre validation. L'hypothèse est devenue postulat!

\section{Le risque calculé}

11 Comme nous venons de le voir, il y a des risques à considérer la présomption d'incompétence comme inéluctable. Mais il faut reconnaître que cette incompétence « appréhendée » est l'une des variables qui entrent dans la réflexion de celui qui songe à promouvoir l'un de ses collaborateurs. Il serait, sans doute, commode de trouver tous les éléments qui modifient ce qu'on perçoit comme la compétence d'une personne dans un poste. On pourrait ainsi évaluer les risques présents chez un individu et renforcer, par une formation d'appoint ou un encadrement plus serré, les éléments les plus fragiles de sa personnalité. Certains de ces éléments sont déjà bien identifiés. Ils font partie des habiletés techniques (comptabilité) ou stratégiques (planification) du gestionnaire. Par contre, il est à la fois plus difficile d'identifier et de corriger une incompétence reliée à ce qu'on pourrait appeler des éléments tactiques faisant partie de la gestion quotidienne.

L'un de ces éléments tactiques concerne les communications du nouveau cadre avec ses collaborateurs immédiats, l'ensemble de l'organisation et son milieu socio-économique (clients, fournisseurs, gouvernements). On a déjà abondamment parlé des attitudes qu'il fallait adopter pour développer la motivation des membres d'une organisation. Mais il s'agit toujours d'améliorations actives qui "projettent» la personnalité du cadre vers l'extérieur. Qu'il s'agisse d'adopter une image de "transparence» ou de favoriser le 
« dialogue », on mise toujours sur l'action et l'initiative de celui qui veut améliorer « ses » communications.

On oublie que le nouveau cadre n'est pas seulement un émetteur de communications; il en est aussi le récepteur. Il n'est pas le seul à produire des messages dans l'organisation. Il en reçoit de toutes parts. On doit donc s'intéresser non seulement à l'état actuel des communications du candidat, mais aussi à l'habileté qu'il pourra avoir à réagir au nombre de communications dont il sera le récepteur dans l'exercice de ses fonctions. Il ne s'agit plus ici seulement de la qualité des communications, mais de leur quantité !

\section{Le temps de réaction}

Pour mieux comprendre ce phénomène, nous allons comparer le réseau de communication d'une organisation à un circuit électrique. Le cadre serait ainsi comme un corps par lequel doit passer le courant électrique de communications. Il le reçoit et le retransmet sous forme de réactions modulées par les compétences techniques et stratégiques dont nous avons parlé plus haut.

on sait que tous les corps présentent une certaine opposition au passage du courant électrique. Cette opposition a été identifiée et définie en 1827 par Georges Simon Ohm et est appelée « la résistance électrique ». De même, le membre de l'organisation peut offrir plus ou moins de résistance à la réception des communications. Cela dépend de l'identification de ses propres buts à ceux de l'organisation et de ses relations interpersonnelles avec son entourage.

La résistance concerne donc la capacité d'une personne à intégrer dans sa pensée ou son action le contenu d'un message organisationnel et sa volonté d'y donner suite. «Je ne fais pas ce que mon superviseur me demande parce que je le considère incompétent.» Ou encore, "Je vais résister à l'introduction d'un ordinateur dans mon département parce que cela va m'enlever toute marge de manœuvre dans l'accomplissement de mes tâches. » Par contre, on est moins familier avec une autre forme d'opposition très particulière qui se manifeste seulement lorsque le courant qui circule dans un circuit VARIE. Tirant ses origines de phénomènes magnétiques étudiés par Lenz, cette opposition est appelée « réactance inductive ».

18 Poursuivons notre métaphore. La réactance, dans l'organisation, serait une résistance, non plus à un message, mais à une quantité de messages. On pourrait parler, en d'autres termes, d'un temps de réaction plus ou moins long aux différentes communications auxquelles on est soumis. Si la réactance est faible, on a l'habitude de se laisser influencer par une série de communications différentes et discontinues. On réagit rapidement à toutes les modifications de messages. C'est le cas, par exemple, d'une téléphoniste ou d'une préposée au comptoir de service. Dans ces fonctions, la réactance DOIT être faible ; le temps de réaction doit être court. L'activité est modulée par une série de messages qui demandent une attention et une réaction immédiates.

Mais si la réactance est élevée, on préfère réagir lentement à un petit nombre de communications. Par exemple, la directrice des achats n'a pas la même réactance que la vendeuse de plancher.

Si la vendeuse est promue directrice des achats, pour-ra-t-elle modifier sa réactance ? Ne risque-t-elle pas de chercher à maintenir son niveau de réactance en réagissant trop vite 
ou en multipliant artificiellement les communications? Habituée à régler son activité sur les demandes qu'elle recevait de la part des clients ou les instructions de son gérant, elle a besoin de cette «drogue " pour continuer à fonctionner. Elle va chercher des excuses pour venir «sur le plancher » ou, encore, multiplier les conversations téléphoniques et les réunions pour assurer sa diète quotidienne de communications.

C'est aussi le drame de ces nouveaux superviseurs qui envient le rythme de travail de leurs anciens collègues. Ils ne peuvent s'empêcher de continuer à faire des tâches qu'ils devraient dorénavant déléguer à d'autres. Ils limitent ainsi la marge de manœuvre de leurs employés, créent un climat d'insécurité par leurs interventions intempestives et les corrections qu'ils se croient obligés d'opérer dans leurs tâches quotidiennes. Tout cela nuit à la motivation du personnel et distrait le cadre de ses responsabilités propres d'organisation à moyen et long termes du travail.

\section{La « réactance » progressive}

On pourrait dire que la réactance doit augmenter à mesure qu'on monte dans un organigramme de type classique. Le cadre intermédiaire doit établir les objectifs des membres de son équipe et en vérifier la réalisation. S'il réagit à chacune des actions posées, il en oubliera la coordination de l'ensemble. S'il accède à un poste de cadre supérieur, il devra, encore une fois, allonger son temps de réaction, et donc augmenter sa réactance, pour tenir compte des effets à long terme de chacune de ses décisions.

L'incompétence serait, dans ce contexte, causée par une incapacité à modifier la sensibilité qui convenait parfaitement à un poste inférieur. Si le nouveau cadre ne résiste pas suffisamment à la tentation de réagir trop vite aux différentes communications qui lui parviennent (réactance trop faible), il risque d'imprimer à son organisation une série de mouvements désordonnés incompatibles avec une solide planification de ses orientations. Il en arrivera même à initier des communications dans le seul but d'avoir la quantité de «feedback » dont sa boulimie communicationnelle ne saurait se passer. Il se mettra à l'écoute de toutes les communications, sans tenir compte des pouvoirs délégués qui l'entourent. Il serait d'ailleurs tentant d'y voir la source de certaines conversions surprenantes au bien-fondé d'une politique de "porte ouverte» ou d'une stratégie de gestion que les théoriciens ont appelé "management by walking» (gestion en se promenant dans l'entreprise).

\section{Du Principe à la pratique}

$\mathrm{Au}$ lieu de se croire condamné à subir la fatalité du fameux Principe, le gestionnaire aurait donc avantage à évaluer, d'abord, son propre niveau de réponse à la quantité de communications. Il peut se demander si son appétit d'informations quotidiennes ne trahit par une réelle insécurité devant la réflexion à moyen et long termes que l'entreprise attend de lui. Habitué à réagir rapidement aux informations qu'il recevait dans un poste inférieur, n'est-il pas en train de recréer cette situation sous prétexte d'être "près de l'action »? N'est-il pas en train de condamner ses collaborateurs à un simple rôle de courroie de transmission? Or c'est justement cette absence de marge de manœuvre ou d'un certain pouvoir de décision qui détruit la motivation des cadres intermédiaires et les entraîne à développer des réflexes bureaucratiques. Ils sont ainsi poussés à multiplier les 
actions délinquantes leur permettant de conserver des bribes d'information ou de créer des informations parasites pour distraire leur patron.

L'accélération des moyens de communication, dont l'informatique est la plus récente locomotive, semble justifier une sensibilité accrue envers toutes sortes d'informations de la part des gestionnaires. Il est vrai que tous ces instruments permettent d'élargir et d'accélérer la cueillette, le «stockage » et la consultation des informations. Mais il y a risque que la quantité ne fasse oublier la qualité de ces informations, surtout si cette quantité répond à la nervosité du gestionnaire concerné. Contrairement à l'analogie habituelle, ce serait un cas où « la forêt cache l'arbre » dont la sève ou le bois pourraient nourrir la réflexion ou soutenir la décision. La compétence sera jugée en fonction de l'habileté à choisir l'information pertinente et, plus précisément, à concilier les diverses informations dans une perspective correspondant aux responsabilités croissantes imposées par la progression hiérarchique du candidat.

Afin d'éviter l'application du Principe de Peter, le cadre supérieur doit donc favoriser les techniques de traitement de l'information et laisser à ses collaborateurs le soin de veiller à la cueillette et au "stockage». Une étude sérieuse de ses propres habitudes lui permettra de déceler les symptômes d'une réaction inadéquate. Cet examen s'applique aussi bien à lui-même qu'à ses collaborateurs immédiats.

Ce que nous venons de dire à propos de l'informatique illustre bien les effets du niveau de sensibilité du cadre sur l'utilisation de toutes les techniques de communication, du journal d'entreprise aux réunions hebdomadaires. Car le niveau de compétence est intimement lié à la "vision " large ou étroite de celui à qui on demande d'assumer une part de plus en plus grande de la survie et du développement de l'entreprise. Si les connaissances et les habiletés professionnelles (dans le sens large du terme) justifient la promotion d'un cadre, le Principe de Peter ne pourra jouer qu'en cas de dysfonction entre cette «vision » et les pratiques quotidiennes du gestionnaire. C'est l'ajustement de ces pratiques que la résistance aux communications permet d'évaluer. Après avoir fait l'éloge de la rapidité de réaction du gestionnaire " compétent » pour faire face aux changements de son marché, ne faudrait-il pas lui rappeler qu'il a aussi une obligation de réflexion, c'est-à-dire de pause et de sélection entre l'information et la réaction?

\section{BIBLIOGRAPHIE}

Hull, Raymond, The Peter Principle : why things always go wrong, N.Y., William Morrow, 1969. Trad.

fr. Le prìncipe de Peter, Paris, Le livre de poche, 1973.

Parkinson, Cyril Northcote, Parkinson's law or the poursuit of progress, N.Y., John Murray, 1958. Cité, en exergue, dans la version de poche publiée par Bantam.

Sheid, Jean-Claude, Les grands auteurs en organisation, Paris, Dunod, 1980. pp. 60-65.

Bernoux, Philippe, La sociologie des organisations, coll. Points, Paris, Seuil, 1985. pp. 193-194.

Lazarus, Richard S., Psychological stress and the Coping Process, N.Y., McGraw-Hill, 1971. 


\section{RÉSUMÉS}

Le célèbre Principe de Peter affirme que « dans une hiérarchie, un employé tend à s'élever à son niveau d'incompétence ». Cette incompétence est l'une des variables qui entrent dans la réflexion de celui qui songe à promouvoir l'un de ses collaborateurs. Il tente de la mesurer en essayant d'anticiper que le collaborateur pourra avoir à réagir au nombre de communications dont il sera le récepteur dans l'exercice de ses nouvelles fonctions.

Le futur cadre peut offrir plus ou moins de résistance à la réception des communications. Il peut aussi manifester une réactance, dans l'organisation, serait une résistance, non plus à un message, mais à une quantité de messages. On pourrait parler, en d'autres termes, d'un temps de réaction plus ou moins long aux différentes communications auxquelles la personne est soumise. Si la réactance est faible, on a l'habitude de se laisser influencer par une série de communications différentes et discontinues. Elle réagit rapidement à toutes les modifications de messages. Si la réactance est élevée, elle préfère réagir lentement à un petit nombre de communications. Nous pensons que la réactance doit augmenter à mesure qu'on monte dans un organigramme de type classique. L'incompétence proviendrait, dans ce contexte, d'une incapacité à modifier la sensibilité qui convenait parfaitement à un poste inférieur.

The famous Peter Principle states that « within an organisation an employee will rise to the level of his own incompetence. » This incompetence is one of the variables which have to be taken into consideration by anyone who is thinking of promothing a colleague.

He tries to measure this by attempting to anticipate that his colleague might have to react to a number of communications, which he might receive while carrying out his new duties, the future executive might resist these communications to a greater or lesser degree. He might develop a more or less resistant reaction not just to one message but to a number of messages. In other words, we can talk about different reaction times to the different messages that might be received.

If the resistance reaction is low, it is because a person is used to being influenced by a series of different and discontinuous communications That person reacts quickly to any changes in the messages.

If resistance is strong the person perfers to react slowly to a small number of communications. In our opinion, the resistance increases as a person moves higher in a classic-type organisation. In this context, incompetence arises from an inability to modify that sensitivity which turned out to be perfectly adapted in a lower job.

\section{AUTEUR}

\section{ANDRÉ LAFRANCE}

André A. Lafrance est professeur au Département de communication de l'Université de Montréal et directeur du Groupe d'intervention stratégique en communication organisationnelle (GISCOR). 